\title{
ÉCHANGES TRANSCULTURELS CHEZ MONIQUE PROULX ET INÊS PEDROSA
}

\author{
Licia Soares de Souza ${ }^{1}$
}

\begin{abstract}
Résumé: Cette étude examine comment deux écrivaines, l'une du Québec, l'autre du Portugal/Brésil, collaborent à la féminisation de la littérature contemporaine par le prisme de leurs réflexions sur certains déplacements historico-symboliques, tout en mettant en lumière le formidable réseau de savoirs qui circulent dans ces espaces du continent américain depuis la colonisation. La première écrivaine que nous présentons est Monique Proulx, auteure de Ce qu/il reste de moi (2015). La deuxième est Inês Pedrosa, d'origine portugaise, journaliste et auteure de plusieurs récits pour enfants, qui a milité contre la criminalisation de l'avortement et pour le mariage de personnes du même sexe. En 2008, elle a publié le roman A Eternidade e o Desejo (L'Éternité et le Désir) qui se déroule dans la ville de Salvador, première capitale du Brésil, qu'elle avait visitée quelques années auparavant. Proulx et Pedrosa abordent un imaginaire de mouvance, tout en mettant en évidence une multiplicité de trajectoires qui fonctionnent comme des connexions contradictoires et des croisements de narratives formant des espaces toujours en conflit. Elles font ressortir, de la sorte, les ambivalences des écritures des mouvements migrants qui ont marqué les métissages issus des rencontres différenciées sur les territoires des Amériques. Proulx évoque les textes de La Folle Entreprise, de Jeanne Mance comme texte fondateur qui prône pour la convivialité des cultures différenciés dans le Nouveau Monde, et Pedrosa utilise les textes du Père Vieira pour montrer des enseignements de savoir-vivre entre les autochtones et les colons dans la Nouvelle Lusitanie. Elles montrent comment la fiction fait jouer les figures renouvelées de certaines utopies du Nouveau Monde dans les textes littéraires, textes qui témoignent d'une puissante facture féminine pour porter un regard neuf sur l'histoire.
\end{abstract}

Mots-clés: Folle entreprise. Monique Proulx, Inês Pedrosa. Jeanne Mance. Antonio Vieira.

\section{TRANSCULTURAL ECHANGES ABOUT MONIQUE PROULX AND INES PEDROSA}

\begin{abstract}
This text depicts how two writers, one from Quebec, Monique Proulx, the other from Portugal/Brazil, Inês Pedrosa, contribute to the feminization of the contemporary literature. They discuss some socio-historical displacements, showing the fantastic web of knowledges that circulates in the spaces of the

\footnotetext{
${ }_{1}$ Professora permanente no Programa de Pós-Graduação em Crítica Cultural, do Campus 2 da UNEB. Professora associada da Universidade do Québec em Montréal. Pesquisadora do CNPQ. Membro do grupo de pesquisa e criação La Traversée que trabalha segundo os princípios metodológicos da Geopoética. Endereço eletrônico: liciasos@hotmail.com
} 
American continent since colonization. Monique Proulx talks about The Crazy Enterprise; the project of solidarity conceived by Jeanne Mance, one of the founders of Montreal. It would bring together French Colonials and Native Americans in a harmonic life to develop the New France" with peace, harmony and collaboration. Even though this project didn't evolve as its thinkers foresaw, it fructified in the minds of people from Montreal, the desire to share the city with several different cultures. On the other hand, Pedrosa shows how it is possible to traverse the streets and view the monuments of Salvador, the first capital of Brazil, reading and commenting on the texts of Father Antonio Vieira. He also tried to understand the life of Natives as an important part of the New World configuration. These two writers are then two significant feminist voices of modern literature that are enable to energize the transcultural work of the women.

Keywords: Monique Proulx. Inês Pedrosa. Transculturalism. Feminism. Crazy Enterprise.

Ce texte examine comment deux écrivaines, l'une du Québec, l'autre du Portugal/Brésil, collaborent à la féminisation de la littérature contemporaine par le prisme de leurs réflexions surcertains déplacements historico-symboliques, tout en mettant en lumière le formidable réseau de savoirs qui circulent dans ces espaces du continent américain depuis la colonisation.

La première écrivaine est Monique Proulx. Native du Québec, elle a obtenu un baccalauréat en littérature et en théâtre de l'Université Laval. Devenue professeur de français et animatrice de théâtre, elle s'est orientée plus intensivement vers l'écriture. Sa première publication est un recueil de nouvelles intitulé « Sans cœur et sans reproche » (1983). En 1996, elle a signé un deuxième recueil de nouvelles: «Les Aurores montréales». Elle est également l'auteur de romans comme "Le Sexe des étoiles» (1987), qui est adapté au cinéma. Elle a été honorée à maintes reprises pour ses œuvres, notamment « Sans cœur et sans reproche " pour laquelle elle a reçu en 1983 le prix Adrienne-Choquette et le prix littéraire Desjardins.

La deuxième est Inês Pedrosa, d'origine portugaise, qui a obtenu une licence en communication à l'Université Nouvelle de Lisbonne. Journaliste et auteure de plusieurs récits pour enfants, et de chroniques féministes, elle a milité contre la criminalisation de l'avortement et pour le mariage de personnes du même sexe. Elle a été la leadeuse de la Marche de la fierté 
Gay em 2005, à Lisbonne. En 2008, elle a publié le roman $A$ Eternidade $e$ o Desejo (L'Éternité et le Désir) qui se déroule dans la ville de Salvador, première capitale du Brésil, qu'elle avait visitée quelques années auparavant.

Proulx et Pedrosa abordent un imaginaire de mouvance, tout en mettant en évidence une multiplicité de trajectoires qui fonctionnent comme des connexions contradictoires, souvent inespérées, et des croisements de narratives formant des espaces toujours en conflit. Elles soulignent l'instabilité des formations sociales, grâce à l'asymétrie entre l'imaginaire culturel et la mobilité des réseaux historiques. Elles font ressortir, de la sorte, les ambivalences des écritures des mouvements migrants qui ont marqué les métissages issus des rencontres différenciées sur les territoires des Amériques.

Commençons notre approche du roman Ce qu'il reste de moi (2015), de Proulx, dans lequel la romancière entend montrer plusieurs visages de Montréal comme espace urbain particulier du Nouveau Monde. Pour ce faire, elle donne vie au personnage Laurel qui, fasciné, par les récits de Jeanne Mance au sujet de son projet de la "Folle Entreprise », entre 1642 et 1665, décide d'écrire un autre récit sur le Montréal contemporain qui pourrait être un reflet de ce projet du temps de la Nouvelle France. Celui-ci repose sur un rêve qui profile le Nouveau Continent:avec le soutien de grands donateurs privés, ils érigeraient une ville "mixte », au sein de laquelle les Européens immigrés et les Amérindiens devenus chrétiens vivraient ensemble, dans un climat d'harmonie et de solidarité. Proulx déclare qu'un tel projet de cohabitation harmonieuse n'a jamais été mis en œuvre, bien entendu, mais qu'il a nourri pendant des siècles une représentation dans l'esprit des Montréalais. Celle de la construction d'une cité où ses nouveaux habitants, les immigrants venus des quatre coins du monde, trouveraient un espace idéal d'accueil pour vivre en communion avec les natifs.

Pour Laurel, la ville est un être vivant qui enfle, se dilate, change de visage en fonction des événements historiques, sociaux, politiques et culturels depuis sa fondation, avec une mémoire qui gagne à être connue par ces nouveaux habitants. Cette quête des origines est toutefois critiquée par son père Thomas, un cinéaste de renom, qui ne peut pas comprendre pourquoi il 
faudrait écrire sur des faits historiques, dans un siècle déjà dominé par une technologie performante.

Laurel insiste, toutefois, pour mettre en relief l'histoire de la ville marquée par les échanges culturels entre les colons et les Amérindiens. Jeanne Mance a fondé un hôpital, où elle a servi comme infirmière, habitée par sa vision de rêve d'un « un paradis terrestre touffu, des chênes, des hêtres, des cèdres, des peupliers... » (PROULX, 2015, p. 11). Le croisement du texte historique et du récit fictionnel vient refléter ainsi le raccourci d'une traversée du passé impliquant des mutations dans les références aux archives historiques, dans la mesure où l'écrivain ouvre la porte à une actualisation successive des aspects différenciés de l'expérience de la fondation d'une cité. La matérialisation explicite de l'espace-charnière de l'écriture de Laurel signifie que la problématique abordée concerne moins le besoin de montrer la dénotation du fait historique et la perpétuation des mythes fondateurs nationaux, que, plutôt, la question de l'actualisation d'une mémoire collective qui est plurielle à sa naissance. Laurel ne veut pas non plus saisir la configuration de la métropole québécoise comme sémiosphère de l'usage de la haute technologie, comme le pense son père, mais interroger le passé historique (la zone mixte, celle de la Folle Entreprise de Jeanne Mance) pour déchiffrer le temps présent d'un espace-charnière qui peut faciliter la convivialité de plusieurs peuples.

D'un autre côté, dans l'Éternité et le Désir, Clara, le personnage principal est une Portugaise, qui retourne au Brésil, après y avoir vécu une expérience tragique, quelques années auparavant. Dans une fête populaire, elle s'est retrouvée au milieu d'un échange de tirs, et, blessée par une balle, elle a perdu la vue. Cette fois-ci, elle est guidée par un ami, Sebastião, qui traverse avec elle les différents lieux de la ville de Salvador, en lui expliquant la signification de ses territoires différenciés. Clara engage sa narration, en affirmant qu'elle va faire d'un espace physique un espace symbolique par le truchement des yeux de son ami, en estimant que les «mots demeurent » et les « couleurs s'évanouissent», dans un parcours qui est plus auditif que visuel. Ce parcours semble prendre une direction métonymique, fonctionnant comme une sorte de traversée du Portugal dans son ancienne 
colonie, exactement pour voir comment elle s'est reconfigurée après son indépendance.

Mais si les yeux de Sebastião secondent Clara pour donner un corps auditif et tactile aux mots que celle-ci doit décoder pour interpréter sa traversée dans la vieille capitale, ils seront aussi susceptibles de féconder les idées qu'elle rumine pour rapprocher une temporalité historique et une temporalité mythique autour d'une appropriation symbolique d'un territoire d'identité transculturelle. Clara projette les points de vue du Père Antonio Vieira sur l'espace qu'elle traverse. Ce jésuite, célébré par l'immense poète Fernando Pessoa "Empereur de la prose portugaise », a peu à peu développé, d'abord auprès de correspondants ecclésiastiques, puis dans différents ouvrages, une interprétation renouvelée du mythe sébastianiste (et des prophéties de Bandarra), dans laquelle il annonce l'avènement futur d'un Quint-Empire (Cinquième Empire) universel dominé par le Portugal sous l'inspiration duSaint-Esprit, après la résurrection du roi Jean IV. Cet Empire fonctionnerait comme la Folle Entreprise de Jeanne Mance: à savoir une perspective d'union harmonieuse entre les colons et les Amérindiens. Vieira avait une autre perception de ces derniers que la Compagnie de Commerce de Portugal avec le Brésil. Il ne ménage pas ses critiques au régime de l'esclavage africain, tout en faisant part d'idées neuves sur la Compagnie de Commerce entre le Portugal et le Brésil.

C'est avec cet arrière-plan historique que Clara visite les églises barroques et les temples sacrés du candomblé, qu'elle évoque l'interdépendance des formations culturelles qui émanent de ces lieux vénérables. Comme Laurel, elle recourt aux mémoires transocéaniques, pour indiquer comment un faisceau de relations s'est installé au sein d'un territoire pour dessiner un monde hybride qui s'est bâti à partir de la rencontre de peuples différents. Le rituel est le langage des rencontres et jaillit tout entier de ce lien entre le ressenti et le geste. Des connexions vont alors s'opérer entre les diverses forces en jeu, l'univers de la foi catholique et celui des dieux africains, et elles vont permettre à la narratrice aveugle de façonner son propre univers symbolique, un univers cénesthésique axé sur le pouvoir de nomination des mots. Le syncrétisme religieux exige la description des fusions entre la conception doloriste du martyre des saints 
catholiques et la perception joyeuse des mouvements des danses colorées et rythmées lors de la descente des dieux dans des personnes bien réelles. Et Clara doit ainsi réussir à révéler la conscience du nouvel homme brésilien déchiré entre ces appels contradictoires: d'un côté la terre ancestrale qui incarne l'ordre et la permanence et de l'autre les espaces rebelles des peuples autrefois asservis, qui sont autant de gages de vitalité et de liberté.

Un exemple emblématique de cette fusion transculturelle est ce qu'elle nomme "la douceur spécifique de la civilisation portugaise», un métissage créatif, qui mettait un baume sur les cruautés et les souffrances de la réalité inhumaine de l'esclavage. Ce sont les fêtes de la Confrérie de Notre Dame de la Bonne Mort qui ont lieu dans la ville de Cachoeira près de la capitale, Salvador. C'est d'ailleurs à Cachoeira qu'a surgi une sorte de Jeanne d'Arc brésilienne, Maria Quitéria, qui prit part à la bataille finale pour l'Indépendance du pays, déguisée en soldat.

Pour Clara, le premier mouvement féministe du Brésil voit le jour au sein de cette Confrérie de la Bonne Mort, fondée en 1820, par des femmes noires, esclaves affranchies remises en liberté, qui ont fondu leur dévotion à Notre Dame, héritage des Jésuites, dont la statue a été immédiatement mise aux côtés des dieux forgés en cuivre ou taillés dans le bois, de facture animiste et fétichiste. Ce miracle du métissage, avait été annoncé par Vieira, qui n'a cessé d'utiliser dans ses écrits, le verbe « luzir » [luire, briller, éclairer, répandre de la lumière]. La colonie portugaise serait une Nouvelle Lusitanie ${ }^{1}$, qui serait prête à briller, à répandre sa clarté dans plusieurs directions.

Revenons à Ce qu'il reste de moi. Dans cette même perspective de réflexion sur les passages transculturels fertiles, dans une sorte d'espacecharnière, Proulx nous donne les clefs pour faire ressortir l'intensité des échanges lors de l'apprentissage de la langue. Sa tante Gaby enseigne le français à des migrants d'origines différentes. La salle de cours se présente comme un kaléidoscope de toutes les cultures : les musulmanes avec ou sans foulard, les Africaines excisées, les Roumaines exploitées par des souteneurs, les petites Coréennes épuisées par les mauvais boulots, les SudAméricaines poursuivies par l'Immigration, les Indiens de la caste des brahmanes, les vieux Haïtiens. On commence ici à apprendre à s'unir autour d'un besoin commun, celui de maîtriser les codes socioculturels 
de la société d'accueil. Observons ce fragment de cours pour saisir le caractère discontinu et progressif de l'acculturation des immigrants :

Aujourd'hui, on célèbre la lettre P....Pakir et Pino, par pitié, ne papotez plus...Des fruits?...Patate, bouh, c'est une légume, ça Barbara, papaya, excellent, pomme, bien sûr, pamplemousse, bravo, Tonio, pinapel, Lily?...Tu veux dire ananas...Prune, poire, pastèque, pruneau...(PROULX, 2015, p. 213).

...Des métiers maintenant...Ppp...Plombier, oui!Papa, ah, ah! Policier, hélas, oui. Professeur! À qui le dites-vous?, et dur métier à part ça. Photographe, très, très bien. Pape! Pourquoi pas?''adore ça, pape, et le féminin est? Papesse!...Pharaon?...Excellent!...Qui a dit Pharaon?(PROULX, 2015, p. 215)

À travers ces bribes de mots et de phrases, ces allitérations avec la lettre $\mathrm{P}$, l'auteure pointe du doigt l'apprentissage de la langue par des êtres circulant à la périphérie de la société urbaine, mais qui font des efforts considérables pour passer au centre, y occuper des positions énonciatrices déterminantes.

Quant à Clara, elle cherche toujours, dans les extraits des écrits de Vieira, des motivations pour continuer à se muer en constructrice de mots susceptibles de rendre compte de la multi-perspective du baroque colonial qui continuerait à alimenter l'histoire des relations diverses entre les différentes cellules de formation de ce monde nouveau. La cécité est pour elle une révolution, de celles qui dévoilent le caractère palpable des mots. Et n'a-t-elle pas trouvé dans le Sermon de Notre Dame des Ó, une description plastique de la lettre $\mathrm{O}$, dénotant l'idée d'un colonie qui progresse grâce à ses propres roues, les roues du temps, qui luisent et transforment les pensées les plus étroites, et qui permettent de fascinants voyages à travers la densité symbolique inscrite dans le nouveau monde qui émerge ? Bien consciente de l'inexistence de paysages plastiques qui puissent caractériser la colonie portugaise avec des couleurs et des contours précis, elle veut montrer l'extraordinaire diversité des récits qui peuvent émaner des mots, en tant que fruits des différents processus d'appropriation et de mélanges qui ont marqué le paysage culturel, de facture américaine, de la nation brésilienne au cours des siècles. 
L'écriture de Monique Proulx, à la fois fluide et complexe, proclame haut et fort son appartenance à une littérature de facture historique, avec un volet de référence féminine. On y trouve des canevas intertextuels qui vont des hypotextes coloniaux à ceux qui décrivent l'histoire des migrations aux $\mathrm{XX}^{\text {ème }}$ et $\mathrm{XXI}^{\text {ème }}$ siècles. Des substrats théoriques - narratifs, génériques ou culturels - innervent une fiction savante qui récupère l'importance historique de la figure de Jeanne Mance comme co-fondatrice de Montréal, contrariant même au passage les documents officiels. Les mises en abîme historiques témoignent de l'arrivée et de la vie de Jeanne Mance à Montréal. Quand elles sont mises en relation, par ce que nous avons caractérisé comme des espaces-charnières, elles sont capables de brouiller les frontières et d'agencer une multiplicité d'images ou des fragments de langages en des séquences uniques pouvant fonctionner comme des espaces-gigognes.

Telle est notre dynamique d'observation. Les espaces-charnières fonctionnent comme des passerelles pour instaurer le règne de la contingence et du hasard, à savoir la défaite de tout sens. Proulx est capable, dans Ce qu'il reste dans moi, de regrouper plusieurs identités éclatées, qui se trouvent ainsi a-sémiotisées, autour du projet d'une convivialité désirée dès la fondation de la ville. Mais Montréal, par sa croissance effrénée, erratique, imprévisible, parle comme ville, comme centre de la grande sémiosphère québécoise, tout en montrant ses collages parataxiques, fruits de la difficile intégration entre des personnages aux origines et conditions sociales diverses. C'est dans ce sens qu'on parle d'espaces-gigognes qui sont aptes à emboîter plusieurs espaces et temporalités, comme un horizon qui abriterait d'autres horizons. Le déroulement diachronique des idées historiques transocéaniques de Jeanne Mance qui viennent se brancher sur les univers contemporains, se mâtine ainsi d'un héritage discursif fermement revendiqué qui vient revamper, reconstruire ou réinventer tout un ensemble de formations sociales et culturelles mettant en valeur un protagonisme féminin lors de la fondation de Montréal.

Ainsi en est-il de l'écriture de Pedrosa. Même si sa source d'inspiration est un homme, un religieux portugais, la jeune femme aveugle met en œuvre une mémoire puissante qui rappelle les principes dynamiques de la convivialité despeuplesqui se sont 
rencontrés sur un territoire du Nouveau Monde. Ce n'est pas un hasard si son guide s'appelle Sebastião, métaphore du jeune roi disparuau cours de la bataille d'Alcácer-Quibir contre les Saadiens, mais qui est demeuré dans l'imaginaire des Portugais et des Brésiliens l'espoir de la reconstruction d'un monde nouveau fait d'abondance. Ils traversent des espaces hybrides, des espaces-charnières, guidés par la voix de l'Empereur de la prose portugaise. Cette traversée produit aussi ses espaces-gigognes, ses gisements mystiques, qui vont nourrir des assemblages de langages variés dans des territoires consacrés où s'expriment librement les chants et les rites des femmes de la trempe des guerrières qui ont lutté conte l'esclavage.

Proulx produit également des gigognes, ses gisements mystiques, avec des vécus différenciés: la synagogue où prie l'hasside, le Quartier des spectacles où l'artiste donne une performance, le Centre Bell où la foule crie, galvanisée par un but des Canadiens, plusieurs histoires de vie enfouies sous les artères de Montréal, depuis que Jeanne Mance s'y est installée il y a plus de quatre cents ans et y a laissé son cœur. Il y a de la diffraction ici aussi, avec la force d'un projet esthétique associé à l'ouvrage et à sa construction. Toutefois, la trame historique de Laurel vient assurer une ligature de sens, et montrer que le rêve utopique d'une société «mixte», qui existe depuis la fondation de Montréal, peut être capable de réunir des peuples différenciés dans cette sémiosphère urbaine.

$\mathrm{Au}$ fond, la structure narrative des deux romans suit un cheminement similaire. On avance du contact vers l'emmagasinement de références, des charnières aux gigognes. La narrativité se fait transmission, les jeux de voix et les métissages des langages parviennent à révéler une réalité complexe, qui permet l'interculturel, mais pointe les difficultés d'intégration des cultures différenciées encore présentes dans les sociétés visées. Salvador, première capitale du Brésil, bâtie avec la sueur des esclaves, continue d'affronter des problèmes d'exclusion sociale. Quelques centaines d'années plus tard, Ville-Marie - devenue Montréal - abrite des hommes et des femmes qui se battent, chacun à sa façon, pour redresser les fortifications d'une vie en proie à diverses vicissitudes, mais rencontre également des problèmes d'exclusion/inclusion. C'est ainsi que ces écrivaines, Proulx et Pedrosa donnent vie à une dynamique de l'imaginaire, une activité 
d'interaction entre des confluences dialogiques spécifiques. Elles montrent comment la fiction fait jouer les figures renouvelées de certaines utopies du Nouveau Monde dans les textes littéraires, textes qui témoignent d'une puissante facture féminine pour porter un regard neuf sur l'histoire.

\section{Références}

PEDROSA, Inês. A eternidade e o desejo. Rio de Janeiro, Objetiva, 2008.

PROULX, Monique. Ce qu'il reste de moi. Montréal, Boréal, 2015.

SOUZA, Licia Soares de, “Representações de Salvador na literatura brasileira contemporânea: por um modelo de geopoética urbana." In: Pontos de Interrogação, Salvador (UNEB), v. 8, n. 1, jan.-jun., p. 153-176, 2018. file:///C:/Users/Licia\%20Casa/Downloads/5201-13960-2-PB\%20(2).pdf, site consulté le 23 avril 2019.

SOUZA, Licia Soares de. Pour une geopoétique interaméricaine. Paris, Société des Écrivains, 2019.

SOUZA, Licia Soares de. Utopies américaines au Québec et au Brésil. Les Presses de l'Université Laval, 2004, (col. Américana).

ALVARES, Sônia Carbonell. Educação estética para jovens e adultos. In: $V$ Seminário Nacional de Formação de Educadores de Jovens e Adultos. Campinas: Unicamp, 2015.

ARAÚJO, Cristiane Brígida de Melo. A educação na Prisão: Reflexões acerca da EJA no processo de ressocialização. 2013. 22 f. TCC (Graduação) - Curso de Ciências Biológica, Universidade Estadual da Paraíba, Campina Grande - PB, 2013.

BOBBIO, Norberto. A era dos direitos. 7a Reimp. Trad. Carlos Nelson Coutinho. Rio de Janeiro: Elsevier, 2004.

BONDIA, Jorge Larossa. Notas sobre a experiência e o saber de experiência. In:

Seminário Internacional de Educação de Campinas. Campinas, 2002.

BRASIL. Lei $n^{\circ}$ 7210, de 11 de julho de 1984, Institui a Lei de Execução Penal. Diário Oficial da União. Brasília - DF, 1984.

BRASIL. Constituição da República Federativa do Brasil. Brasília - DF, 1988.

BRASIL. Lei de Diretrizes e Bases da Educação Nacional (LDB) $n^{0}$ 9394/96. Brasília, 1996. 
ÉCHANGES TRANSCULTURELS CHEZ MONIQUE PROULX ET INÊS PEDROSA

BRASIL. Parecer CEB № 11/2000. Diretrizes Curriculares Nacionais para a Educação de Jovens e Adultos. Brasília, 2000.

BRASIL. Lei 12.289. Dispõe sobre a criação da Universidade da Integração Internacional da Lusofonia Afro-Brasileira - UNILAB e dá outras providências. Brasília, 2010.

Recebido em 12 de maio de 2019.

Aceito em 01 de junho de 2019. 